\title{
Lung Assist Devices Influence Cardio-Energetic Parameters: Numerical Simulation Study
}

\author{
C. De Lazzari, B. Quatember, W. Recheis, M. Mayr, S. Demertzis, G. Allasia, A. De Rossi, R. \\ Cavoretto, E. Venturino, I. Genuini
}

\begin{abstract}
We aim at an analysis of the effects mechanical ventilators (MVs) and thoracic artificial lungs (TALs) will have on the cardiovascular system, especially on important quantities, such as left and right ventricular external work (EW), pressure-volume area (PVA) and cardiac mechanical efficiency (CME). Our analyses are based on simulation studies which were carried out by using our CARDIOSIM ${ }^{\odot}$ software simulator. At first, we carried out simulation studies of patients undergoing mechanical ventilation (MV) without a thoracic artificial lung (TAL). Subsequently, we conducted simulation studies of patients who had been provided with a TAL, but did not undergo MV. We aimed at describing the patient's physiological characteristics and their variations with time, such as EW, PVA, CME, cardiac output (CO) and mean pulmonary arterial/venous pressure (PAP/PVP). We were starting with a simulation run under well-defined initial conditions which was followed by simulation runs for a wide range of mean intrathoracic pressure settings. Our simulations of MV without TAL showed that for mean intrathoracic pressure settings from negative $(-4 \mathrm{mmHg})$ to positive $(+5$ mmHg) values, the left and right ventricular EW and PVA, right ventricular $\mathrm{CME}$ and $\mathrm{CO}$ decreased, whereas left ventricular CME and the PAP increased. The simulation studies of patients with a TAL, comprised all the usual TAL arrangements, viz. configurations "in series" and in parallel with the natural lung and, moreover, hybrid configurations. The main objective of the simulation studies was, as before, the assessment of the hemodynamic response to the application of a TAL. We could for instance show that, in case of an "in series" configuration, a reduction (an increase) in left (right) ventricular EW and PVA values occurred, whereas the best performance in terms of $\mathrm{CO}$ can be achieved in the case of an in parallel configuration.
\end{abstract}

\section{INTRODUCTION}

Mechanical ventilators (MVs) and thoracic artificial lungs (TALs) are being applied to assist patients affected by

C. D. is with the National Institute for Cardiovascular Research (INRCBologna) and with the Institute of Clinical Physiology UOS of Rome (CNR), Via S.M. della Battaglia, 4400185 Rome Italy (corresponding author e-mail: claudio.delazzari@ ifc.cnr.it).

B. Q. is with the Medizinische Universitaet Innsbruck, Austria.

W. R. is with the Univ.-Klinik für Radiologie, Innsbruck Medical University, Austria.

M. M is with the University of Applied Sciences Wiener Neustadt, Austria.

S. D. is with the Cardiocentro Ticino, Lugano, Switzerland.

G. A.; A. D.; R. C.; E. V. are with the Dipartimento di Matematica, Università degli Studi di Torino, Italy.

I. G. is with Department of Cardiovascular, Respiratory, Nephrological, Anesthesiological and Geriatric Sciences, "Sapienza" University of Rome Italy. respiratory failure. In the following, we will investigate specific aspects of their use in clinical practice.

Mechanical Ventilation (MV). Generally, the treatment with a ventilator is mandatory whenever a patient's respiratory system becomes unable to keep the concentrations of $\mathrm{O}_{2}$ and $\mathrm{CO}_{2}$ in blood at tolerable levels. There exists a whole range of clinical applications of MV. Severe impairments of the lungs may occur in serious cases of chronic obstructive pulmonary disease (COPD), acute respiratory distress syndrome (ARDS), and in pneumonia. A ventilator may also be necessary for patients who need ventricular assist device (VAD) assistance [1]. MV of the respiratory system gives rise to specific interactions with other organs. These interactions may cause complications and other adverse effects. In particular, MV has a significant effect on the cardiovascular system [2]. We will focus here on these interactions and quantitatively describe the effects of MV on the cardiovascular system by carrying out simulation studies with our simulator CARDIOSIM ${ }^{\mathbb{O}}$ which will be described in detail later. By using CARDIOSIM $^{(}$, we simulated these interactions for different modes of operation. In particular, we determined specific energetic and hemodynamic quantities and parameters of the cardiovascular system influenced by MV, such as left and right ventricular external work $(\mathrm{EW})$, pressure-volume area (PVA), cardiac mechanical efficiency (CME) [3], cardiac output $(\mathrm{CO})$, and mean pulmonary arterial pressure. MVs are widely used. A whole variety of forms and types of these lung assist devices (LADs) exists. In the past, anesthetists have also made use of our simulation facilities to acquire data which allowed them to study and compare the performance capacities and especially the effects the ventilators will have on the cardiovascular system [4],[5].

Thoracic Artificial Lung (TAL). TAL can be regarded as a viable alternative to $\mathrm{MV}$. This lung assist device (LAD) is for instance advantageously used as an interim aid for patients who are waiting on a lung transplant, since, on the one hand, mechanical ventilation would be a contraindication for lung transplantation and, on the other hand, a TAL has the ability to either partially or totally fulfill the function of natural lungs. In the following, we will deal with simulation studies which we carried out by using our simulator CARDIOSIM $^{\odot}$. We designed a special module for TAL [6],[7] and configured CARDIOSIM ${ }^{\mathcal{C}}$ for our investigations in a straightforward manner by appropriately interconnecting the already existing individual software modules and the newly-developed module for the TAL. The TAL can be arranged either in parallel or "in series" with the natural lung. Moreover, a hybrid mode is also possible. 


\section{MATERIAL AND METHOD}

\section{A. Numerical simulator}

We will aim again at characterizing the interactions between LAD and the cardiovascular system. In doing so, we carried out simulation studies with our numerical simulator CARDIOSIM ${ }^{\odot}$. As already adumbrated above, CARDIOSIM $^{\odot}$ is hallmarked by its modular building principle. A library of lumped components (modules) has been created, the most important modules of them are:

Modules "Left Heart" and "Right Heart." These (software) modules comprise mathematical models describing the behavior of the ventricles, the atria and the septa. For the ventricles, atria and the ventricular septum we used a variable elastance modeling approach, whereas we assumed that the atrial septum as being rigid [8],[9].

Module "Systemic Arterial Section." In our modeling approach, the systemic arterial section is described by three individual resistance-inertance-compliance (RLC) lumped elements AT, TT and ABT (Windkessel-like models [10]); AT describes the arterial, TT the thoracic and ABT the abdominal part of the systemic circulation (Fig. 1).

$>$ Modules "Systemic Venous Section" and "Pulmonary Venous Section." Each of these venous sections is described by one resistance-compliance (RC) lumped element [11].

Module "Main Pulmonary Artery Section." This module describes the larger pulmonary arteries by one resistanceinertance-compliance (RLC) lumped element (Windkessellike modeling approach [6],[7],[12]).

> Module "Small Pulmonary Artery Section." This module describes all the small intra-pulmonary arteries proximal to the arterioles by one resistance-inertance-compliance (RLC) lumped element (Windkessel-like modeling approach [7],[12]).

> Module "Pulmonary Arterioles Section." This section was modeled by only a single resistance, since the arterioles are relatively stiff, and, moreover, the inertial forces are negligible [7],[12].

Module "Pulmonary Capillaries Section." This section has justifiably also been modeled by a single resistance [7],[12].

$>$ Module "Coronary Circulation." This section was modeled as a lumped parameter model based on the intramyocardial pump concept. For a more detailed description, please refer to [10].

Module “Thoracic Artificial Lung” This module is based on a lumped parameter modeling approach by using resistance - inertance - compliance (RLC) elements [6],[7]. Of particular importance are the resistances (variables) $\mathrm{R}_{\text {TALan, }}, \mathrm{R}_{\text {TALala }}$ and $\mathrm{R}_{\text {TALpab. }}$ By appropriately assigning finite or infinite values (cut off) to them, we are able to configure the "Module TAL" for "in series", parallel or hybrid mode of operation (Fig. 1).
The modular building principle of CARDIOSIM ${ }^{\odot}$ allows us to assemble a simulation system which is tailored to the given problem area simply by appropriately interconnecting these modules. Fig. 1 depicts the way of assembling the above-described modules for our simulation studies of the effects of the lung assist devices on the cardiovascular system.

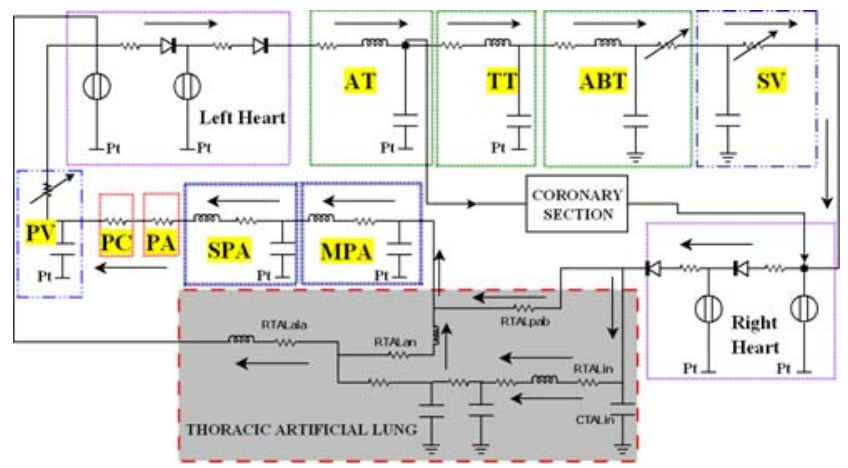

Figure 1. Electric analogue of the cardiovascular system with TAL. AT, TT and ABT represent the aortic, thoracic and abdominal tract, respectively. $\mathrm{SV}(\mathrm{PV})$ is the systemic (pulmonary) venous section. MPA (SPA) is the main (small) pulmonary artery section. PA (PC) is the pulmonary arteriole (capillary) section. Pt is the mean intrathoracic pressure. $R_{\text {TALin }}\left(C_{\text {TALin }}\right)$ is the inlet graft resistance (compliance) to the TAL. $\mathrm{R}_{\text {TALala }}$ and $\mathrm{R}_{\text {TALpab }}$ are two resistances used to divide the flow between the pulmonary circulation $\left(\mathrm{R}_{\text {TALpab }}\right)$ and the left atrium ( $\left.\mathrm{R}_{\text {TALala }}\right) . \mathrm{R}_{\text {TALan }}$ is the outlet graft resistance to the pulmonary artery.

In Fig. $1 \mathrm{Pt}$ is the mean intrathoracic pressure and it is defined as:

$$
P t=\frac{1}{T} \int_{0}^{T} p_{t}(t) d t
$$

where $\mathrm{T}$ is the duration of a ventilatory cycle and $\mathrm{p}_{\mathrm{t}}(\mathrm{t})$ an instantaneous value of the time-varying thoracic pressure.

\section{B. Experimental method}

As already mentioned earlier, our simulation studies comprise two kinds of studies, viz. (a) investigations of the interactions between MV (without TAL) and the cardiovascular system and (b) investigations of the interactions between the application of a TAL (without MV) and the cardiovascular system. In both kinds of investigations, there are methodological similarities and differences [13]:

Similarities. In both kinds of investigations, we carried out several simulation runs, each for a distinct value of the varying quantity Pt. The results reveal the varying effect of the respiratory system on the cardiovascular system [5].

Particularities - MV without TAL. To assess the effect of MV on the cardiovascular system, we started with a simulation run at baseline conditions ( $\mathrm{Pt}=0 \mathrm{mmHg}$ ); afterwards we carried out simulation runs for varying levels of $\mathrm{Pt}$ ranging from $-4 \mathrm{mmHg}$ to $+5 \mathrm{mmHg}$ in steps of 1 $\mathrm{mmHg}$.

Particularities - TAL without MV. As before, we aim again at the effect this lung assist device will have on the cardiovascular system. Our simulation studies comprised 
TALs "in series", parallel and hybrid mode of operation. Simulation runs were carried out for each mode of operation at two levels of $\mathrm{Pt}$, viz. $\mathrm{Pt}=0 \mathrm{mmHg}$ and $\mathrm{Pt}=-4 \mathrm{mmHg}$, which we will regard as being two different baseline conditions. The configuration of TAL for the "in series" mode of operation is effected by assigning the infinite value (cut off) to the resistances $R_{\text {TALpab }}$ and $R_{\text {TALala, whereas a finite value is }}$ assigned to the resistance $\mathrm{R}_{\text {TALan }}$. In contrast, the configuration of TAL for the parallel mode of operation is effected by assigning the infinite value (cut off) to the resistance $R_{\text {TALan, }}$, whereas finite values are assigned to the resistances $R_{\text {TALpab }}$ and $R_{\text {TALala }}$. However, in hybrid mode of operation, finite values are given to all the aforementioned resistances $R_{\text {TALan, }} R_{\text {TALpab }}$ and $R_{\text {TALala }}$ (Fig. 1). Our simulation studies comprised simulation runs for varying values of $\mathrm{R}_{\mathrm{TALin}}$ (from 2 to $700 \mathrm{~g} \cdot \mathrm{cm}^{-4} \cdot \mathrm{sec}^{-1}$ ) and $\mathrm{C}_{\mathrm{TALin}}$ (from 0.5 to $\left.5 \mathrm{~cm}^{3} \cdot \mathrm{mmHg}^{-1}\right)$.

\section{RESULTS}

The majority of the results of our simulation studies will be presented in graphical form so that their interpretation will be easy and quick. Moreover, only such types of graphical representation will be used which could provide physicians with a deep understanding of the consequences an envisaged respiratory assistance strategy may have for the patient's cardiovascular system.

MV without TAL. The simulation results comprise the following parameters of the cardiovascular system: left heart and right heart PVA, EW, CME, CO and mean pulmonary arterial (venous) pressure PAP (PVP). These parameters have been acquired by carrying out simulation runs for changing values of $\mathrm{Pt}$ from $-4 \mathrm{mmHg}$ to $5 \mathrm{mmHg}$ in steps of $1 \mathrm{mmHg}$. The values of the aforementioned parameters at $\mathrm{Pt}=0 \mathrm{mmHg}$ are regarded as being baseline values. It is appropriate to express the parameter values which we obtained by carrying out the simulation runs for all other values of $\mathrm{Pt}$ as percentage of the baseline values. These "percentage changes values" are graphically represented in the four column diagrams of Fig. 2. It can easily be seen that for negative (positive) values of $\mathrm{Pt}$ :

$\checkmark$ Cardiac output CO, left (right) EW and PVA, will show an increase (a reduction) of their values in terms of percent with respect to baseline values, whereas

$\checkmark$ PAP and PVP will show a decrease (increase) of their values, likewise in terms of percent with respect to baseline values.

$\underline{T A L}$ without $M V$. The first series of simulation runs referred to patients whose respiratory functions are impaired to such an extent that they rely on the assistance of a TAL. We assumed that the patient is breathing quietly, and Pt will vary between $-4 \mathrm{mmHg}$ (inspiration phase) and $0 \mathrm{mmHg}$ (expiration phase). The simulation studies include the three aforementioned modes of operations of the TAL, viz. the in parallel, "in series", and hybrid mode. Primarily for comparison reasons we included in these studies also the case of quiet breathing conditions without TAL. Moreover, we confined the simulation runs to the aforementioned two distinct values of the mean intrathoracic pressures, $\mathrm{Pt}=-4$ $\mathrm{mmHg}$ and $\mathrm{Pt}=0 \mathrm{mmHg}$.
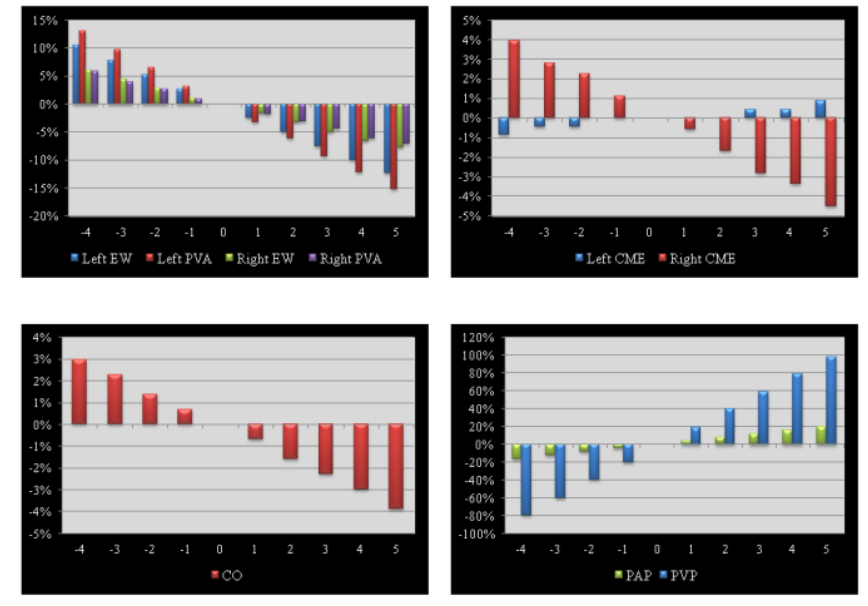

Figure 2. Percentage changes calculated respect to $\mathrm{Pt}=0 \mathrm{mmHg}$ when the effects of MV was reproduced. The two upper panels show the percentage changes for the left and right ventricular energetic variables. The lower panel show the percentage changes of cardiac output (left panel) and of PAP and PVP.

This first series of simulations is based on the assumption that the applied TAL is modeled by using the module "Thoracic Artificial Lung" with the following specifications: $\mathrm{R}_{\mathrm{TALin}}=20 \mathrm{~g} \cdot \mathrm{cm}^{-4} \cdot \mathrm{sec}^{-1}$ and $\mathrm{C}_{\mathrm{TALin}}=0.9 \mathrm{~cm}^{3} \cdot \mathrm{mmHg}^{-1}$ (Fig. 1). The results are graphically represented in Fig. 3 as four column diagrams showing the values of the physiological parameters left EW (PVA) and right EW (PVA). The results of the first series of simulation runs refer to breathing without a TAL and with a TAL having the (fixed) values $\mathrm{R}_{\text {TALin }}=20$ $\mathrm{g} \cdot \mathrm{cm}^{-4} \cdot \mathrm{sec}^{-1}$ and $\mathrm{C}_{\mathrm{TALin}}=0.9 \mathrm{~cm}^{3} \cdot \mathrm{mmHg}^{-1}$. The acquired hemodynamic parameters EW and PVA which are visualized as column diagrams in Fig. 3 will allow experienced clinicians and especially novices to attain an overview of the hemodynamic consequences of the application of a TAL. This first series of simulations was continued by a second and third series of simulation runs. These simulation runs have primarily been carried out to complement the knowledge which has already been derived from the first series of simulations, especially by studying to what extent deviations from the above values of $\mathrm{R}_{\mathrm{TALin}}=20 \mathrm{~g} \cdot \mathrm{cm}^{-4} \cdot \mathrm{sec}^{-1}$ and $\mathrm{C}_{\mathrm{TALin}}=$ $0.9 \mathrm{~cm}^{3} \cdot \mathrm{mmHg}^{-1}$ will affect the hemodynamic parameters.

In particular, the aim of the second series of simulation studies is to study the hemodynamic consequences a TAL will have on the left and right ventricular quantities EW and PVA, and, moreover, on the parameters $\mathrm{CO}$ and PAP in cases where the resistance RTALin deviated from its previously fixed value of $\mathrm{R}_{\mathrm{TALin}}=20 \mathrm{~g} \cdot \mathrm{cm}-4 \cdot \mathrm{sec}-1$, whereas the compliance $\mathrm{C}_{\text {TALin }}=0.9 \mathrm{~cm}_{3} \cdot \mathrm{mmHg}_{1}$ remained unchanged. We carried out simulation runs for the wide range of RTALin from 2 to 700 $\mathrm{g} \cdot \mathrm{cm}-4 \cdot \mathrm{sec}-1$. For each value of RTALin, the simulation runs involved all the three modes of operation ("in series", parallel, and hybrid), and, as before, we confined the simulation runs to the two distinct values of the mean intrathoracic pressures, $\mathrm{Pt}=-4 \mathrm{mmHg}$ and $\mathrm{Pt}=0 \mathrm{mmHg}$. 

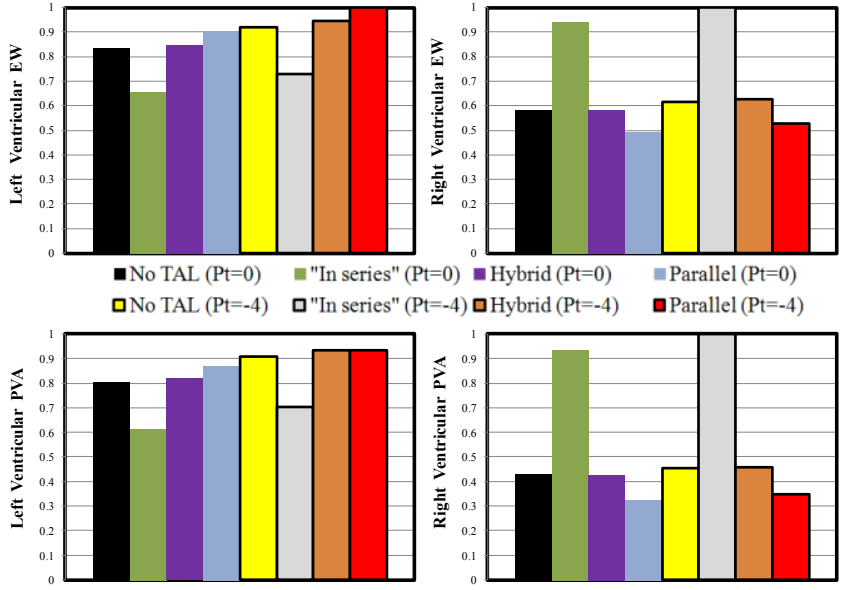

Figure 3. Left and right ventricular EW and PVA without and with TAL assistance. The simulations were performed setting $\mathrm{Pt}=-4$ and $\mathrm{Pt}=0 \mathrm{mmHg}$.

TAL assistance was applied "in series", in parallel and in hybrid mode.

For each panel data were normalized to the maximum value. Inlet graft resistance and compliace were se to $\mathrm{R}_{\mathrm{TALin}}=20\left[\mathrm{~g} \cdot \mathrm{cm}^{-4} \cdot \mathrm{sec}^{-1}\right]$ and $\mathrm{C}_{\mathrm{TALin}}=0.9$ $\left[\mathrm{cm}^{3} \cdot \mathrm{mmHg}^{-1}\right]$

Fig. 4 depicts the variations of the ventricular energetic parameters left EW (PVA) and right EW (PVA) with the value of $R_{\text {TALin }}$ which is assumed to rise from 2 to 700 $\mathrm{g} \cdot \mathrm{cm}^{-4} \cdot \mathrm{sec}^{-1}$, whereas the variations of the hemodynamic parameters $\mathrm{CO}$ and PAP with rising $\mathrm{R}_{\mathrm{TALin}}$ can be seen in Fig. 5 (diagrams in the lower row). In Figs. 4 and 5, the ordinate scales are percentage scales; in all diagrams the percentage expresses how large or small the values of the individual hemodynamic quantities (with TAL) are relative to the corresponding quantities in case of quiet breathing conditions without TAL (baseline conditions). It can be seen in Fig. 4 that, roughly speaking, the left (right) ventricular EW and PVA increase (decrease) with rising values of $R_{\text {TALin }}$. Moreover, the values of left (right) ventricular EW and PVA for the "in series" mode of operation are essential higher than those in case of the parallel and hybrid mode. In contrast, the influence of the value of $\mathrm{Pt}$ on the just mentioned quantities is almost insignificant. Fig. 5 (lower row of diagrams) shows that the hemodynamic parameter $\mathrm{CO}$ increases with rising values of $R_{\text {TALin }}$; the influence of the value of Pt is again almost insignificant. The diagram in the lower right-hand corner of Fig. 5 shows that, roughly speaking, the mean PAP increases with rising $\mathrm{R}_{\text {TALin }}$ if TAL is applied in "in series" mode. In the case of parallel and hybrid mode both parameters, $\mathrm{CO}$ and PAP, are only changing insignificantly with rising values of $R_{\text {TALin }}$ up to $R_{\text {TALin }}=150 \mathrm{~g} \cdot \mathrm{cm}^{-4} \cdot \mathrm{sec}^{-1}$ and then decrease significantly for higher values of $\mathrm{R}_{\mathrm{TALin}}$. Fig. 5 (diagrams in the upper row) depicts the variations of the hemodynamic parameters $\mathrm{CO}$ and mean $\mathrm{PAP}$ with rising values of $\mathrm{C}_{\mathrm{TALin}}$. In particular, the value of $\mathrm{CO}$ increases with rising values of $\mathrm{C}_{\text {TALin }}$ if the TAL is applied in the "in series" mode of operation, whereas in this mode the values of mean PAP are barely changed.

The third series of simulation runs aims at the hemodynamic consequences a TAL will have on the left and right ventricular quantities EW and PVA, and, moreover, on the parameters $\mathrm{CO}$ and PAP in cases where the compliance $\mathrm{C}_{\mathrm{TALin}}$ deviated from its previously fixed value of $\mathrm{C}_{\mathrm{TALin}}=0.9$ $\mathrm{cm}^{3} \cdot \mathrm{mmHg}^{-1}$, whereas the resistance $\mathrm{R}_{\mathrm{TALin}}=20 \mathrm{~g} \cdot \mathrm{cm}^{-4} \cdot \mathrm{sec}^{-1}$ remained unchanged (Figs. 5 and 6).

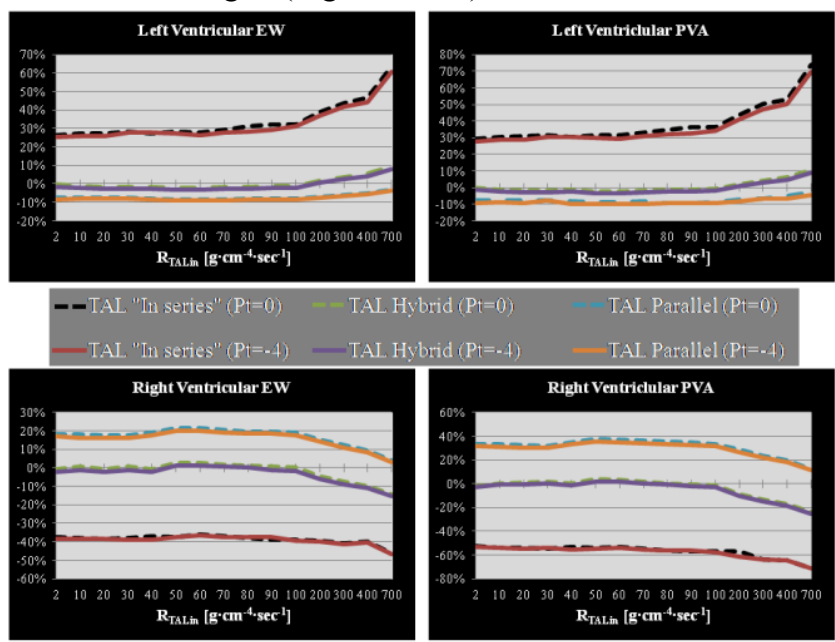

Figure 4. Percentage changes of left and right ventricular EW and PVA. During the different simulations $\mathrm{C}_{\text {TALin }}$ was set to $0.9\left[\mathrm{~cm}^{3} \cdot \mathrm{mmHg}^{-1}\right]$. The simulations were performed setting $\mathrm{Pt}=-4$ and $\mathrm{Pt}=0 \mathrm{mmHg}$. TAL assistance was applied "in series", in parallel and in hybrid mode.

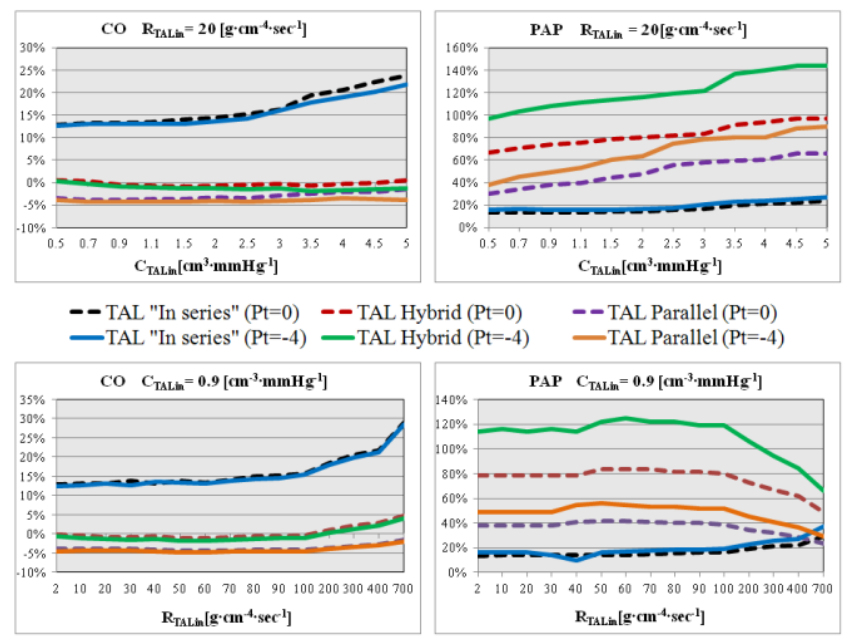

Figure 5. Percentage changes of $\mathrm{CO}$ and mean PAP. The simulations were performed setting $\mathrm{Pt}=-4$ and $\mathrm{Pt}=0 \mathrm{mmHg}$. TAL assistance was applied "in series", in parallel and in hybrid mode. Percentage changes were calculated with respect to baseline conditions without TAL assistance.

In particular, we carried out simulation runs for the wide range of $\mathrm{C}_{\text {TALin }}$ from 0.5 to $5.0 \mathrm{~cm}^{3} \cdot \mathrm{mmHg}^{-1}$. For each value of $\mathrm{C}_{\mathrm{TALi}}$, the simulation runs involved all the three modes of operation ("in series", parallel and hybrid), and, as before, we confined the simulation runs to the two distinct values of the mean intrathoracic pressure, viz. $\mathrm{Pt}=-4 \mathrm{mmHg}$ and $\mathrm{Pt}=0$ mmHg. In Figs. 5 and 6, the ordinate scales are, as before, percentage scales. In these diagrams, the percentage expresses again how large or small the values of the individual hemodynamic quantities (with TAL) are relative to the corresponding quantities in case of quiet breathing conditions without TAL (baseline conditions). Fig. 6 depicts the variations of the energetic parameters left and right 
ventricular $\mathrm{EW}$ and $\mathrm{PVA}$ with rising values of $\mathrm{C}_{\mathrm{TALin}}$ (range of 0.5 to $5.0 \mathrm{~cm}^{3} \cdot \mathrm{mmHg}^{-1}$ ).

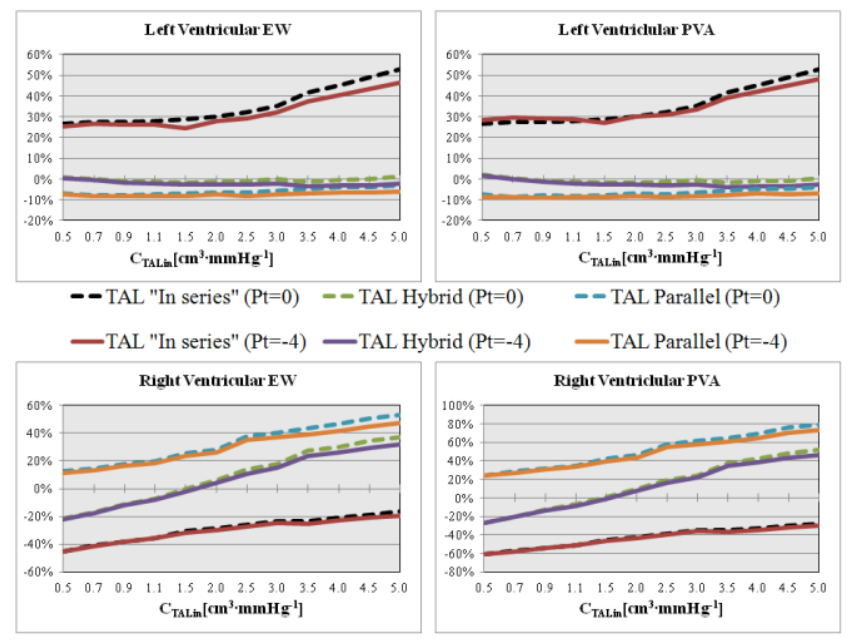

Figure 6. Percentage changes of left and right ventricular EW and PVA. During the different simulations $\mathrm{R}_{\text {TALin }}$ was set to $20\left[\mathrm{~g} \cdot \mathrm{cm}^{-4} \cdot \mathrm{sec}^{-1}\right]$. The simulations were performed setting $\mathrm{Pt}=-4$ and $\mathrm{Pt}=0 \mathrm{mmHg}$. TAL assistance was applied "in series", in parallel and in hybrid mode. Percentage changes were calculated with respect to baseline conditions without TAL assistance.

In the case of "in series" mode of operation, the values of left ventricular EW and PVA increase with rising values of $\mathrm{C}_{\mathrm{TALin}}$. Right ventricular EW and PVA increase (with respect to baseline conditions) even further when TAL assistance was applied in parallel and in hybrid mode.

\section{CONCLUSIONS REMARKS AND FUTURE WORK}

We referred to patients with respiratory failure who will need technical devices to ensure that the concentrations of $\mathrm{O}_{2}$ and $\mathrm{CO}_{2}$ in the blood remain at tolerable levels. All kinds of respiratory assistance have severe consequences on other parts of the human body, especially on the circulation. We investigated the influence of the respiratory support on the entire cardiovascular system by carrying out simulation studies with our newly-developed software simulator CARDIOSIM $^{\odot}$. Unfortunately, the current status of our modeling approach does not sufficiently take into account the details of the complicated anatomic structure of the cardiovascular system; and, moreover, it does not describe the markedly nonlinear characteristics of the cardiovascular system; furthermore, the assumption of a uniform intrathoracic pressure must be regarded as being a considerable simplification. Hence, the simulation results attained with our model can only be regarded as relatively coarse approximations. Nonetheless, it proved that the results of our present simulation studies have provided clinicians with valuable insights on how to quantitatively assess the effects of assisted respiration on the cardiovascular system. Furthermore, CARDIOSIM ${ }^{\odot}$ facilitated important clinical decision-making processes. Yet, we endeavor to improve our simulation modeling concept, primarily (a) by arranging for a more detailed description of the anatomy by using an essentially higher number of lumped components, (b) by taking in full account the nonlinear mechanical behavior of the vessel walls, and (c) by a more sophisticated characterization of the variations of the extramural pressure of the blood vessels throughout the human body (intrathoracic, intrapulmonary, and intraabdominal pressure). This future work will be done step by step. In each step, the model will be validated.

\section{REFERENCES}

[1] Y. Ochiai, P. M. McCarthy, N. G. Smedira, M. K. Banbury, J. L. Navia, J. Feng, A. P. Hsu, M. L. Yeager, T. Buda,K. J. Hoercher, M. W. Howard, M. Takagaki, K. Doi, K. Fukamachi, "Predictors of severe right ventricular failure after implantable left ventricular assist device insertion: Analysis of 245 patients", Circulation, vol. 106, suppl. I, pp. I-198-I-202, 2002.

[2] J. R. Mitchell, R. Sas, D. J. Zuege, C. J. Doig, E. R. Smith, W. A. Whitelaw, J. V. Tyberg, I. Belenkie, "Ventricular interaction during mechanical ventilation in closed-chest anesthetized dogs", Can. J. Cardiol., vol. 2, no. 1, pp. 73-81, January 2005.

[3] K. Sagawa, L. Maughan, H. Suga, K. Sunagawa, Cardiac contraction and the pressure-volume relationships, New York: Oxford University Press, 1988.

[4] C. De Lazzari, M. Darowski, P. Wolski, G. Ferrari, G. Tosti, DM Pisanelli, "In vivo and simulation study of artificial ventilation effects on energetic variables in cardiosurgical patients", Methods Inf Med, vol. 44, no. 1, 98-105, 2005.

[5] C. De Lazzari, M. Darowski, G. Ferrari, D.M. Pisanelli, G. Tosti, "Modelling in the study of interaction of Hemopump device and artificial ventilation", Computers in biology and medicine, vol. 36, no. 11, pp. 1235-1251, 2006.

[6] F. Boschetti, C. E. Perlman, K. E. Cook, L. F. Mockros, "Hemodynamic effects of attachment modes and device design a thoracic artificial lung", ASAIO J., vol. 46, no. 1, pp. 42-48, 2000.

[7] C. De Lazzari, I. Genuini, B. Quatember, F. Fedele, "Mechanical ventilation and thoracic artificial lung assistance during mechanical circulatory support with PUCA pump: In silico study", Computer Methods and Programs in Biomedicine, vol. 113, no. 2, pp. 642-654, February 2014.

[8] W. L. Maughan, K. Sunagawa, K. Sagawa, "Ventricular systolic interdependence: volume elastance model in isolated canine hearts", Am J Physiol. Heart Circ. Physiol., vol. 253, pp. H1381-H1390, 1987.

[9] C. De Lazzari, A. D’Ambrosi, F. Tufano, L. Fresiello, M. Garante, R. Sergiacomi, F. Stagnitti, C.M. Caldarera, N. Alessandri, "Cardiac resynchronization therapy: could a numerical simulator be a useful tool in order to predict the response of the biventricular pacemaker synchronization?", Eur. Rev. Med. Pharmacol. Sci., vol. 14, no. 11, pp. 969-978, 2010.

[10] C. De Lazzari, D. Neglia, G. Ferrari, F. Bernini, M. Micalizzi, A. L'Abbate, M.G. Trivella, "Computer Simulation of Coronary Flow Waveforms during Caval Occlusion", Methods of Information in Medicine, vol. 48, no. 2, pp. 113-122, 2009.

[11] G. Ferrari, C. De Lazzari, R. Mimmo, G. Tosti, D. Ambrosi, "A modular numerical model of the cardiovascular system for studying and training in the field of cardiovascular physiopathology", Journal of biomedical engineering, vol. 14, no.2, pp. 91-107, 1992.

[12] Y. Shi, P. Lawford, R. Hose, "Review of zero-D and 1-D models of blood flow in the cardiovascular system", BioMed. Eng. Online, 10:33 2011, doi:10.1186/1475-925X-10-33.

[13] J. J. Marini, B. H. Culver, L. Butler, "Mechanical effect of lung distension with positive pressure on cardiac function", Am. Rev. Resp. Dis. Vol. 124 pp. 382-386, 1981. 